\title{
Nonexistence of Totally Contact Umbilical Slant Lightlike Submanifolds of Indefinite Cosymplectic Manifolds
}

\author{
Rashmi Sachdeva, ${ }^{1}$ Rakesh Kumar, ${ }^{2}$ and Satvinder Singh Bhatia ${ }^{1}$ \\ ${ }^{1}$ School of Mathematics and Computer Applications, Thapar University, Patiala 147004, India \\ ${ }^{2}$ University College of Engineering, Punjabi University, Patiala 147002, India \\ Correspondence should be addressed to Rakesh Kumar; dr_rk37c@yahoo.co.in
}

Received 1 April 2013; Accepted 12 May 2013

Academic Editors: A. Fino, C. Qu, and E. H. Saidi

Copyright (c) 2013 Rashmi Sachdeva et al. This is an open access article distributed under the Creative Commons Attribution License, which permits unrestricted use, distribution, and reproduction in any medium, provided the original work is properly cited.

We study totally contact umbilical slant lightlike submanifolds of indefinite cosymplectic manifolds. We prove that there do not exist totally contact umbilical proper slant lightlike submanifolds in indefinite cosymplectic manifolds other than totally contact geodesic proper slant lightlike submanifolds. We also prove that there do not exist totally contact umbilical proper slant lightlike submanifolds of indefinite cosymplectic space forms. Finally we give characterization theorems on minimal slant lightlike submanifolds.

\section{Introduction}

The notion of slant submanifolds was initiated by Chen $[1,2]$, as a generalization of both holomorphic and totally real submanifolds in complex geometry. Since then such submanifolds have been studied by many authors. Lotta $[3,4]$ defined and studied slant submanifolds in contact geometry. Cabrerizo et al. studied slant, semislant, and bislant submanifolds in contact geometry $[5,6]$. They all studied the geometry of slant submanifolds with positive definite metric. Therefore this geometry may not be applicable to the other branches of mathematics and physics, where the metric is not necessarily definite. Thus the geometry of slant submanifolds with indefinite metric became a topic of chief discussion, and Şahin [7] played a very crucial role in this direction by introducing the notion of slant lightlike submanifolds of indefinite Hermitian manifolds. Recently Gupta et al. [8] introduced the notion of slant lightlike submanifolds of an indefinite cosymplectic manifold and obtained necessary and sufficient conditions for the existence of slant lightlike submanifolds. In [9], Jain et al. studied GCRlightlike submanifolds of indefinite cosymplectic manifolds and proved that every totally contact umbilical GCR-lightlike submanifold of an indefinite cosymplectic manifold is a totally contact geodesic GCR-lightlike submanifold.
In this paper we prove that there do not exist totally contact umbilical proper slant lightlike submanifolds of indefinite cosymplectic manifolds other than totally contact geodesic. We also prove that there do not exist totally contact umbilical proper slant lightlike submanifolds of indefinite cosymplectic space forms. Finally, we give characterization theorems on minimal slant lightlike submanifolds.

\section{Preliminaries}

An odd-dimensional semi-Riemannian manifold $\bar{M}$ is said to be an indefinite almost contact metric manifold if there exist structure tensors $(\phi, V, \eta, \bar{g})$, where $\phi$ is a $(1,1)$ tensor field, $V$ is a vector field, called structure vector field, $\eta$ is a 1 -form, and $\bar{g}$ is the semi-Riemannian metric on $\bar{M}$ satisfying (see [10]) the following:

$$
\begin{aligned}
& \phi^{2} X=-X+\eta(X) V, \quad \eta \circ \phi=0, \quad \phi V=0, \quad \eta(V)=1, \\
& \bar{g}(\phi X, \phi Y)=\bar{g}(X, Y)-\eta(X) \eta(Y), \quad \bar{g}(X, V)=\eta(X),
\end{aligned}
$$

for $X, Y \in \Gamma(T \bar{M})$, where $\Gamma(T \bar{M})$ denotes the Lie algebra of vector fields on $\bar{M}$. 
An indefinite almost contact metric manifold $\bar{M}$ is called an indefinite cosymplectic manifold if (see [11])

$$
\left(\bar{\nabla}_{X} \phi\right) Y=0, \quad \bar{\nabla}_{X} V=0
$$

for any $X, Y \in \Gamma(T \bar{M})$, where $\bar{\nabla}$ denote the Levi-Civita connection on $\bar{M}$.

Let $(\bar{M}, \bar{g})$ be a real $(m+n)$-dimensional semi-Riemannian manifold of constant index $q$ such that $m, n \geq 1,1 \leq$ $q \leq m+n-1$, let $(M, g)$ be an $m$-dimensional submanifold of $\bar{M}$, and let $g$ be the induced metric of $\bar{g}$ on $M$. If $\bar{g}$ is degenerate on the tangent bundle $T M$ of $M$, then $M$ is called a lightlike submanifold of $\bar{M}$ (see [12]). For a degenerate metric $g$ on $M, T M^{\perp}$ is a degenerate $n$-dimensional subspace of $T_{x} \bar{M}$. Thus both $T_{x} M$ and $T_{x} M^{\perp}$ are degenerate orthogonal subspaces but no longer complementary. In this case, there exists a subspace $\operatorname{Rad} T_{x} M=T_{x} M \cap T_{x} M^{\perp}$ which is known as radical (null) subspace. If the mapping $\operatorname{Rad} T M: x \in$ $M \rightarrow \operatorname{Rad} T_{x} M$ defines a smooth distribution on $M$ of rank $r>0$ then the submanifold $M$ of $\bar{M}$ is called an $r$-lightlike submanifold and $\operatorname{Rad} T M$ is called the radical distribution on $M$.

Screen distribution $S(T M)$ is a semi-Riemannian complementary distribution of $\operatorname{Rad}(T M)$ in $T M$; that is, $T M=$ $\operatorname{Rad} T M \perp S(T M)$ and $S\left(T M^{\perp}\right)$ is a complementary vector subbundle to $\operatorname{Rad} T M$ in $T M^{\perp}$. Let $\operatorname{tr}(T M)$ and $\operatorname{ltr}(T M)$ be complementary (but not orthogonal) vector bundles to $T M$ in $\left.T \bar{M}\right|_{M}$ and to $\operatorname{Rad} T M$ in $S\left(T M^{\perp}\right)^{\perp}$, respectively. Then we have

$$
\begin{aligned}
\operatorname{tr}(T M) & =\operatorname{ltr}(T M) \perp S\left(T M^{\perp}\right) \\
\left.T \bar{M}\right|_{M} & =T M \oplus \operatorname{tr}(T M) \\
& =(\operatorname{Rad} T M \oplus \operatorname{lt}(T M)) \perp S(T M) \perp S\left(T M^{\perp}\right) .
\end{aligned}
$$

For a quasi-orthonormal fields of frames on $T M$, we have the following.

Theorem 1 (see [12]). Let $\left(M, g, S(T M), S\left(T M^{\perp}\right)\right)$ be an $r$ lightlike submanifold of a semi-Riemannian manifold $(\bar{M}, \bar{g})$. Then there exists a complementary vector bundle $\operatorname{ltr}(T M)$ of $\operatorname{Rad} T M$ in $S\left(T M^{\perp}\right)^{\perp}$ and a basis of $\Gamma\left(\left.\operatorname{ltr}(T M)\right|_{u}\right)$ consisting of smooth section $\left\{N_{i}\right\}$ of $\left.S\left(T M^{\perp}\right)^{\perp}\right|_{\mathcal{u}}$, where $u$ is a coordinate neighborhood of $M$ such that

$$
\begin{aligned}
\bar{g}\left(N_{i}, \xi_{j}\right)= & \delta_{i j}, \quad \bar{g}\left(N_{i}, N_{j}\right)=0, \\
& \text { for any } i, j \in\{1,2, \ldots, r\}
\end{aligned}
$$

where $\left\{\xi_{1}, \ldots, \xi_{r}\right\}$ is a lightlike basis of $\Gamma(\operatorname{Rad}(T M))$.

Let $\bar{\nabla}$ be the Levi-Civita connection on $\bar{M}$, then according to the decomposition (5), the Gauss and Weingarten formulas are given by

$$
\bar{\nabla}_{X} Y=\nabla_{X} Y+h(X, Y), \quad \bar{\nabla}_{X} U=-A_{U} X+\nabla_{X}^{\perp} U,
$$

for any $X, Y \in \Gamma(T M)$ and $U \in \Gamma(\operatorname{tr}(T M))$, where $\left\{\nabla_{X} Y\right.$, $\left.A_{U} X\right\}$ and $\left\{h(X, Y), \nabla_{X}^{\perp} U\right\}$ belong to $\Gamma(T M)$ and $\Gamma(\operatorname{tr}(T M))$, respectively. Here $\nabla$ is a torsion-free linear connection on $M$, $h$ is a symmetric bilinear form on $\Gamma(T M)$ which is called the second fundamental form, and $A_{U}$ is a linear operator on $M$ and known as a shape operator.

According to (4), considering the projection morphisms $L$ and $S$ of $\operatorname{tr}(T M)$ on $\operatorname{ltr}(T M)$ and $S\left(T M^{\perp}\right)$, respectively, then (7) becomes

$$
\begin{gathered}
\bar{\nabla}_{X} Y=\nabla_{X} Y+h^{l}(X, Y)+h^{s}(X, Y), \\
\bar{\nabla}_{X} U=-A_{U} X+D_{X}^{l} U+D_{X}^{s} U,
\end{gathered}
$$

where we put $h^{l}(X, Y)=L(h(X, Y)), h^{s}(X, Y)=S(h(X, Y))$, $D_{X}^{l} U=L\left(\nabla_{X}^{\perp} U\right)$, and $D_{X}^{s} U=S\left(\nabla_{X}^{\perp} U\right)$. As $h^{l}$ and $h^{s}$ are $\Gamma(\operatorname{ltr}(T M))$ valued, and $\Gamma\left(S\left(T M^{\perp}\right)\right)$ valued, respectively, therefore they are called as the lightlike second fundamental form and the screen second fundamental form on $M$. In particular

$$
\begin{aligned}
& \bar{\nabla}_{X} N=-A_{N} X+\nabla_{X}^{l} N+D^{s}(X, N), \\
& \bar{\nabla}_{X} W=-A_{W} X+\nabla_{X}^{s} W+D^{l}(X, W),
\end{aligned}
$$

where $X \in \Gamma(T M), N \in \Gamma(\operatorname{ltr}(T M))$ and $W \in \Gamma\left(S\left(T M^{\perp}\right)\right)$. Using (7) and (8) we obtain

$$
\bar{g}\left(h^{s}(X, Y), W\right)+\bar{g}\left(Y, D^{l}(X, W)\right)=g\left(A_{W} X, Y\right),
$$

Let $\bar{P}$ be a projection of TM on $S(T M)$ then using the decomposition $T M=\operatorname{Rad} T M \perp S(T M)$, we can write

$$
\begin{gathered}
\nabla_{X} \bar{P} Y=\nabla_{X}^{*} \bar{P} Y+h^{*}(X, \bar{P} Y), \\
\nabla_{X} \xi=-A_{\xi}^{*} X+\nabla_{X}^{* t} \xi,
\end{gathered}
$$

for any $X, Y \in \Gamma(T M)$ and $\xi \in \Gamma(\operatorname{Rad} T M)$, where $\left\{\nabla_{X}^{*} \bar{P} Y\right.$, $\left.A_{\xi}^{*} X\right\}$ and $\left\{h^{*}(X, \bar{P} Y), \nabla_{X}^{* t} \xi\right\}$ belong to $\Gamma(S(T M))$ and $\Gamma(\operatorname{Rad} T M)$, respectively. Here $\nabla^{*}$ and $\nabla_{X}^{* t}$ are linear connections on $S(T M)$ and $\operatorname{Rad} T M$, respectively. By using (8), (9) and (11), we obtain

$$
\begin{aligned}
\bar{g}\left(h^{l}(X, \bar{P} Y), \xi\right) & =g\left(A_{\xi}^{*} X, \bar{P} Y\right), \\
\bar{g}\left(h^{*}(X, \bar{P} Y), N\right) & =g\left(A_{N} X, \bar{P} Y\right) .
\end{aligned}
$$

\section{Slant Lightlike Submanifolds}

A lightlike submanifold has two distributions, namely, the radical distribution and the screen distribution. The radical distribution is totally lightlike and it is not possible to define angle between two vector fields of radical distribution where the screen distribution is nondegenerate. There are some definitions for the angle between two vector fields in Lorentzian setup [13], but they are not appropriate for our goal. Therefore to introduce the notion of slant lightlike submanifolds, one needs a Riemannian distribution, and Gupta et al. [8] played a crucial role in the development of the theory of slant lightlike submanifolds of indefinite cosymplectic manifolds. 
Definition 2 (see [8]). Let $M$ be an $r$-lightlike submanifold of an indefinite cosymplectic manifold $\bar{M}$ of index $2 r$ with structure vector field $V$ tangent to $M$. Then one say that $M$ is a slant lightlike submanifold of $\bar{M}$ if the following conditions are satisfied.

(a) $\operatorname{Rad} T M$ is a distribution on $M$ such that $\phi \operatorname{Rad} T M \cap$ $\operatorname{Rad}(T M)=\{0\}$.

(b) For all $x \in U \subset M$ and for each non zero vector field $X$ tangent to $\bar{D}=D \perp\{V\}$, if $X$ and $V$ are linearly independent, then the angle $\theta(X)$ between $\phi X$ and the vector space $\bar{D}_{x}$ is constant, where $D$ is complementary distribution to $\phi \operatorname{ltr}(T M) \oplus \phi \operatorname{Rad} T M$ in screen distribution $S(T M)$.

The constant angle $\theta(X)$ is called the slant angle of $\bar{D}$. A slant lightlike submanifold $M$ is said to be proper if $D \neq\{0\}$ and $\theta \neq 0, \pi / 2$.

Since a submanifold $M$ is invariant (anti-invariant, resp.), if $\phi T_{p} M \subset T_{p} M,\left(\phi T_{p} M \subset T_{p} M^{\perp}\right.$, resp. $)$, for any $p \in M$, Therefore from the previous definition, it is clear that $M$ is invariant (anti-invariant, resp.) if $\theta(X)=0(\theta(X)=\pi / 2$, resp.).

Then the tangent bundle $T M$ of $M$ is decomposed as

$$
\begin{aligned}
T M & =\operatorname{Rad} T M \perp S(T M) \\
& =\operatorname{Rad} T M \perp(\phi \operatorname{Rad} T M \oplus \phi \operatorname{ltr}(T M)) \perp \bar{D},
\end{aligned}
$$

where $\bar{D}=D \perp\{V\}$. For any $X \in \Gamma(T M)$, we write

$$
\phi X=T X+F X
$$

where $T X$ is the tangential component of $\phi X$ and $F X$ is the transversal component of $\phi X$. Similarly for any $U \in$ $\Gamma(\operatorname{tr}(T M))$, we write

$$
\phi U=B U+C U,
$$

where $B U$ is the tangential component of $\phi U$ and $C U$ is the transversal component of $\phi U$. Using the decomposition in (13), we denote by $P_{1}, P_{2}, Q_{1}, Q_{2}$, and $\bar{Q}_{2}$ the projections on the distributions $\operatorname{Rad} T M, \phi \operatorname{Rad} T M, \phi \operatorname{tr}(T M), D$ and $\bar{D}=$ $D \perp V$, respectively. Then for any $X \in \Gamma(T M)$, we can write

$$
X=P_{1} X+P_{2} X+Q_{1} X+\bar{Q}_{2} X,
$$

where $\bar{Q}_{2} X=Q_{2} X+\eta(X) V$.

Applying $\phi$ to (16), we obtain

$$
\phi X=\phi P_{1} X+\phi P_{2} X+F Q_{1} X+T Q_{2} X+F Q_{2} X .
$$

Then using (14) and (15), we get

$$
\begin{gathered}
\phi P_{1} X=T P_{1} X \in \Gamma(\phi \operatorname{Rad} T M), \\
\phi P_{2} X=T P_{2} X \in \Gamma(\operatorname{Rad} T M), \\
F P_{1} X=F P_{2} X=0, \quad T Q_{2} X \in \Gamma(D), \\
F Q_{1} X \in \Gamma(\operatorname{ltr}(T M)) .
\end{gathered}
$$

Differentiating (17) and using (8)-(9), (14) and (15), for any $X, Y \in \Gamma(T M)$, we have

$$
\begin{aligned}
\left(\nabla_{X} T\right) Y= & A_{F Q_{1} Y} X+A_{F_{2} Y} X+B h(X, Y), \\
D^{l}\left(X, F Q_{2} Y\right)= & F Q_{1} \nabla_{X} Y-h^{l}(X, T Y)-\nabla_{X}^{l} F Q_{1} Y, \\
D^{s}\left(X, F Q_{1} Y\right)= & F Q_{2} \nabla_{X} Y-h^{s}(X, T Y)+C h^{s}(X, Y) \\
& -\nabla_{X}^{s} F Q_{2} Y .
\end{aligned}
$$

By using cosymplectic property of $\bar{\nabla}$ with (7), we have the following lemmas.

Lemma 3. Let $M$ be a slant lightlike submanifold of an indefinite cosymplectic manifold $\bar{M}$, then on has

$$
\begin{gathered}
\left(\nabla_{X} T\right) Y=A_{F Y} X+B h(X, Y), \\
\left(\nabla_{X}^{t} F\right) Y=C h(X, Y)-h(X, T Y),
\end{gathered}
$$

where $X, Y \in \Gamma(T M)$ and

$$
\left(\nabla_{X} T\right) Y=\nabla_{X} T Y-T \nabla_{X} Y, \quad\left(\nabla_{X}^{t} F\right) Y=\nabla_{X}^{t} F Y-F \nabla_{X} Y .
$$

Lemma 4. Let $M$ be a slant lightlike submanifold of an indefinite cosymplectic manifold $\bar{M}$ then we have

$$
\begin{gathered}
\left(\nabla_{X} B\right) U=A_{C U} X-T A_{U} X, \\
\left(\nabla_{X}^{t} C\right) U=-F A_{U} X-h(X, B U),
\end{gathered}
$$

where $X \in \Gamma(T M)$ and $U \in \Gamma(\operatorname{tr}(T M))$ and

$$
\left(\nabla_{X} B\right) U=\nabla_{X} B U-B \nabla_{X}^{t} U, \quad\left(\nabla_{X}^{t} C\right) U=\nabla_{X}^{t} C U-C \nabla_{X}^{t} U .
$$

Theorem 5. Let $M$ be a slant lightlike submanifold of an indefinite cosymplectic manifold $\bar{M}$; then

(a) the distribution $\bar{D}$ is integrable, if and only if, $h(X, T Y)=h(Y, T X), D^{l}\left(X, F Q_{2} Y\right)=D^{l}\left(Y, F Q_{2} X\right)$, and $\nabla_{X}^{s} F Q_{2} Y=\nabla_{Y}^{s} F Q_{2} X$, for any $X, Y \in \Gamma(\bar{D})$,

(b) the distribution $\phi(\operatorname{lt}(T M))$ is integrable, if and only if $A_{F_{Q_{1}} Y} X=A_{F_{1} X} Y$, for any $X, Y \in \Gamma(\phi(\operatorname{ltr}(T M)))$.

Proof. Using (20) and (21), we have $F \nabla_{X} Y=D^{l}\left(X, F Q_{2} Y\right)+$ $h(X, T Y)+\nabla_{X}^{s} F Q_{2} Y-C h^{s}(X, Y)$, for any $X, Y \in \Gamma(\bar{D})$. Here replacing $X$ by $Y$ and then subtracting the resulting equation from this equation, we get $(A)$. Next from (22) and (23), we have

$$
-T\left(\nabla_{X} Y\right)=A_{F_{1} Y} X+B h(X, Y)
$$

for all $X, Y \in \Gamma(\phi(\operatorname{ltr}(T M)))$. Then, similarly to the above, we have $T[X, Y]=A_{F_{2} X} Y-A_{F_{1} Y} X$, and this completes the proof of $(b)$. 
Corollary 6 (see [8]). Let $M$ be a slant lightlike submanifold of an indefinite cosymplectic manifold $\bar{M}$ with structure vector field $V$ tangent to $M$. Then one has

$$
\begin{aligned}
g & \left(T \bar{Q}_{2} X, T \bar{Q}_{2} Y\right) \\
\quad & \cos ^{2} \theta\left[g\left(\bar{Q}_{2} X, \bar{Q}_{2} Y\right)-\eta\left(\bar{Q}_{2} X\right) \eta\left(\bar{Q}_{2} Y\right)\right], \\
\bar{g} & \left(F \bar{Q}_{2} X, F \bar{Q}_{2} Y\right) \\
\quad & \sin ^{2} \theta\left[g\left(\bar{Q}_{2} X, \bar{Q}_{2} Y\right)-\eta\left(\bar{Q}_{2} X\right) \eta\left(\bar{Q}_{2} Y\right)\right]
\end{aligned}
$$

for any $X, Y \in \Gamma(T M)$.

\section{Totally Contact Umbilical Slant Lightlike Submanifolds}

Definition 7 (see [14]). If the second fundamental form $h$ of a submanifold tangent to characteristic vector field $V$, of an indefinite Sasakian manifold $\bar{M}$ is of the form

$$
\begin{aligned}
h(X, Y)= & \{g(X, Y)-\eta(X) \eta(Y)\} \alpha \\
& +\eta(X) h(Y, V)+\eta(Y) h(X, V),
\end{aligned}
$$

for any $X, Y \in \Gamma(T M)$, where $\alpha$ is a vector field transversal to $M$, then $M$ is called a totally contact umbilical and totally contact geodesic if $\alpha=0$.

The above definition also holds for a lightlike submanifold $M$. For a totally contact umbilical lightlike submanifold $M$, we have

$$
\begin{aligned}
h^{l}(X, Y)= & \{g(X, Y)-\eta(X) \eta(Y)\} \alpha_{L} \\
& +\eta(X) h^{l}(Y, V)+\eta(Y) h^{l}(X, V), \\
h^{s}(X, Y)= & \{g(X, Y)-\eta(X) \eta(Y)\} \alpha_{S} \\
& +\eta(X) h^{s}(Y, V)+\eta(Y) h^{s}(X, V),
\end{aligned}
$$

where $\alpha_{L} \in \Gamma(\operatorname{ltr}(T M))$ and $\alpha_{S} \in \Gamma\left(S\left(T M^{\perp}\right)\right)$.

Lemma 8. Let $M$ be a slant lightlike submanifold of an indefinite cosymplectic manifold $\bar{M}$; then $F Q_{2} X \in \Gamma\left(S\left(T M^{\perp}\right)\right.$ ), for any $X \in \Gamma(T M)$.

Proof. Using (4) and (6), it is clear that $F Q_{2} X \in \Gamma\left(S\left(T M^{\perp}\right)\right)$ if $g\left(F Q_{2} X, \xi\right)=0$, for any $\xi \in \Gamma(\operatorname{Rad}(T M))$. Therefore

$$
\begin{aligned}
\bar{g}\left(F Q_{2} X, \xi\right) & =\bar{g}\left(\phi Q_{2} X-T Q_{2} X, \xi\right) \\
& =g\left(\phi Q_{2} X, \xi\right)=-g\left(Q_{2} X, \phi \xi\right)=0 .
\end{aligned}
$$

Hence the result follows.

Thus from Lemma 8 it follows that $F\left(D_{p}\right)$ is a subspace of $S\left(T M^{\perp}\right)$. Therefore there exists an invariant subspace $\mu_{p}$ of $T_{p} \bar{M}$ such that

$$
S\left(T_{p} M^{\perp}\right)=F\left(D_{p}\right) \perp \mu_{p} .
$$

Thus

$$
\begin{aligned}
& T_{p} \bar{M} \\
& \quad=S\left(T_{p} M\right) \perp\left\{\operatorname{Rad}\left(T_{p} M\right) \oplus \operatorname{ltr}\left(T_{p} M\right)\right\} \perp\left\{F\left(D_{p}\right) \perp \mu_{p}\right\} .
\end{aligned}
$$

Theorem 9. Let $M$ be a totally contact umbilical slant lightlike submanifold of an indefinite cosymplectic manifold $\bar{M}$. Then at least one of the following statements is true:

(i) $M$ is an anti-invariant submanifold,

(ii) $D=\{0\}$,

(iii) if $M$ is a proper slant submanifold, then $\alpha_{S} \in \Gamma(\mu)$.

Proof. Let $M$ be a totally contact umbilical slant lightlike submanifold of an indefinite cosymplectic manifold $\bar{M}$; then for any $X=Q_{2} X \in \Gamma(D)$ with (29), we have

$$
h\left(T Q_{2} X, T Q_{2} X\right)=g\left(T Q_{2} X, T Q_{2} X\right) \alpha .
$$

Therefore from (7), (27), and the above equation, we get

$$
\bar{\nabla}_{\mathrm{TQ}_{2} X} T Q_{2} X-\nabla_{T \mathrm{Q}_{2} X} T Q_{2} X=\cos ^{2} \theta\left[g\left(Q_{2} X, Q_{2} X\right)\right] \alpha .
$$

Using (14) and the fact that $\bar{M}$ is cosymplectic manifold, we obtain

$$
\begin{aligned}
& \phi \bar{\nabla}_{T_{2} X} Q_{2} X-\bar{\nabla}_{T Q_{2} X} F Q_{2} X-\nabla_{T_{2} X} T Q_{2} X \\
& \quad=\cos ^{2} \theta\left[g\left(Q_{2} X, Q_{2} X\right)\right] \alpha .
\end{aligned}
$$

Then using (8) and (9), we get

$$
\begin{aligned}
& \phi \nabla_{T Q_{2} X} Q_{2} X+\phi h^{l}\left(T Q_{2} X, X\right)+\phi h^{s}\left(T Q_{2} X, X\right) \\
& +A_{F Q_{2} X} T Q_{2} X-\nabla_{T Q_{2} X}^{s} F Q_{2} X-D^{l}\left(T Q_{2} X, F Q_{2} X\right) \\
& \quad-\nabla_{T_{2} X} T Q_{2} X=\cos ^{2} \theta\left[g\left(Q_{2} X, Q_{2} X\right)\right] \alpha .
\end{aligned}
$$

Thus using (14), (15), (30), and (31), we have

$$
\begin{aligned}
T \nabla_{T Q_{2} X} Q_{2} X+F \nabla_{T Q_{2} X} Q_{2} X+g\left(T Q_{2} X, X\right) \phi \alpha^{l} \\
\quad+g\left(T Q_{2} X, X\right) B \alpha^{s}+g\left(T Q_{2} X, X\right) C \alpha^{s}+A_{F Q_{2} X} T Q_{2} X \\
\quad-\nabla_{T Q_{2} X}^{s} F Q_{2} X-D^{l}\left(T Q_{2} X, F Q_{2} X\right)-\nabla_{T Q_{2} X} T Q_{2} X \\
=\cos ^{2} \theta\left[g\left(Q_{2} X, Q_{2} X\right)\right] \alpha .
\end{aligned}
$$

Equating the transversal components, we get

$$
\begin{aligned}
& F \nabla_{T Q_{2} X} Q_{2} X+g\left(T Q_{2} X, X\right) C \alpha^{s}-\nabla_{T Q_{2} X}^{s} F Q_{2} X \\
& \quad-D^{l}\left(T Q_{2} X, F Q_{2} X\right)=\cos ^{2} \theta\left[g\left(Q_{2} X, Q_{2} X\right)\right] \alpha .
\end{aligned}
$$


On the other hand, (28) holds for any $X=Y \in \Gamma(D)$, and by taking the covariant derivative with respect to $T Q_{2} X$, we obtain

$$
\bar{g}\left(\nabla_{T_{2} X}^{s} F Q_{2} X, F Q_{2} X\right)=\sin ^{2} \theta g\left(\nabla_{T Q_{2} X} Q_{2} X, Q_{2} X\right) .
$$

Now taking the inner product in (40) with $F Q_{2} X$, we obtain

$$
\begin{aligned}
\bar{g} & \left(F \nabla_{T_{2} X} Q_{2} X, F Q_{2} X\right)-\bar{g}\left(\nabla_{T Q_{2} X}^{s} F Q_{2} X, F Q_{2} X\right) \\
& =\cos ^{2} \theta\left[g\left(Q_{2} X, Q_{2} X\right)\right] \bar{g}\left(\alpha_{S}, F Q_{2} X\right) .
\end{aligned}
$$

Then using (28) and (41), we get

$$
\cos ^{2} \theta\left[g\left(Q_{2} X, Q_{2} X\right)\right] \bar{g}\left(\alpha_{S}, F Q_{2} X\right)=0 .
$$

Thus from (43), it follows that; $\theta=\pi / 2, Q_{2} X=0$ or $\alpha_{S} \in \Gamma(\mu)$. This completes the proof.

Lemma 10. Let $M$ be a totally contact umbilical slant lightlike submanifold of an indefinite cosymplectic manifold $\bar{M}$; then $g\left(\nabla_{X} X, \phi \xi\right)=0$, for any $X \in \Gamma(D)$ and $\xi \in \Gamma(\operatorname{Rad} T M)$.

Proof. Let $X \in \Gamma(D)$; therefore $X=Q_{2} X$, and then using (3), (8), and (9) for a totally contact umbilical slant lightlike submanifold, we have

$$
\begin{aligned}
g\left(\nabla_{X} X, \phi \xi\right) & =\bar{g}\left(\bar{\nabla}_{X} X, \phi \xi\right) \\
& =-\bar{g}\left(\bar{\nabla}_{X} T Q_{2} X, \xi\right)-\bar{g}\left(\bar{\nabla}_{X} F Q_{2} X, \xi\right) \\
& =-\bar{g}\left(h^{l}\left(X, T Q_{2} X\right), \xi\right)-\bar{g}\left(D^{l}\left(X, F Q_{2} X\right), \xi\right) \\
& =-\bar{g}\left(D^{l}\left(X, F Q_{2} X\right), \xi\right),
\end{aligned}
$$

since for $X \in \Gamma(D)$, using (2), (14), and (30), we have $h^{l}(X$, $\left.T Q_{2} X\right)=\left\{g\left(X, T Q_{2} X\right)\right\} \alpha_{L}=0$. Also for $X \in \Gamma(D)$, we have $\eta\left(Q_{2} X\right)=0$, and $\eta(\xi)=0$ therefore by replacing $W$ by $F Q_{2} X$ and $Y$ by $\xi$ in (10) and using the hypothesis that $M$ is totally contact umbilical slant lightlike submanifold, we obtain

$$
\begin{aligned}
\bar{g}\left(D^{l}\left(X, F Q_{2} X\right), \xi\right) & =-\bar{g}\left(h^{s}(X, \xi), F Q_{2} X\right) \\
& =-g(X, \xi) \bar{g}\left(\alpha_{S}, F Q_{2} X\right)=0
\end{aligned}
$$

Therefore from (44) and (45), the result follows.

Theorem 11. Every totally contact umbilical proper slant lightlike submanifold $M$ of an indefinite cosymplectic manifold $\bar{M}$ is totally contact geodesic.

Proof. Since $M$ is a totally contact umbilical slant lightlike submanifold, therefore for any $X=Q_{2} X \in \Gamma(D)$, using (29), we have $h\left(T Q_{2} X, T Q_{2} X\right)=g\left(T Q_{2} X, T Q_{2} X\right) \alpha$, and therefore using (27), we obtain

$$
\begin{aligned}
h & \left(T Q_{2} X, T Q_{2} X\right) \\
& =\cos ^{2} \theta\left[g\left(Q_{2} X, Q_{2} X\right)-\eta\left(Q_{2} X\right) \eta\left(Q_{2} X\right)\right] \alpha \\
& =\cos ^{2} \theta\left[g\left(Q_{2} X, Q_{2} X\right)\right] \alpha .
\end{aligned}
$$

Using (20) for $X \in \Gamma(D)$, we have

$$
\begin{aligned}
F \nabla_{\mathrm{TQ}_{2} X} X= & h\left(T Q_{2} X, T Q_{2} X\right)-C h\left(T Q_{2} X, X\right) \\
& +\nabla_{T Q_{2} X}^{s} F Q_{2} X+D^{l}\left(T Q_{2} X, F Q_{2} X\right) .
\end{aligned}
$$

Since $M$ is totally contact umbilical slant lightlike submanifold therefore $C h\left(T Q_{2} X, X\right)=g\left(T Q_{2} X, X\right) C \alpha=0$, therefore using (46) and (47), we get

$$
\begin{aligned}
& \cos ^{2} \theta\left[g\left(Q_{2} X, Q_{2} X\right)\right] \alpha \\
& \quad=F \nabla_{T Q_{2} X} X-\nabla_{T Q_{2} X}^{s} F Q_{2} X-D^{l}\left(T Q_{2} X, F Q_{2} X\right) .
\end{aligned}
$$

Taking the scalar product of both sides of (48) with respect to $F Q_{2} X$ for $X \in \Gamma(D)$, we obtain

$$
\begin{aligned}
\cos ^{2} & \theta\left[g\left(Q_{2} X, Q_{2} X\right)\right] \bar{g}\left(\alpha_{S}, F Q_{2} X\right) \\
& =\bar{g}\left(F \nabla_{T_{2} X} X, F Q_{2} X\right)-\bar{g}\left(\nabla_{T Q_{2} X}^{s} F Q_{2} X, F Q_{2} X\right) .
\end{aligned}
$$

Here using (28), we get

$$
\begin{aligned}
\cos ^{2} \theta & {\left[g\left(Q_{2} X, Q_{2} X\right)\right] \bar{g}\left(\alpha_{S}, F Q_{2} X\right) } \\
& =\sin ^{2} \theta\left[\bar{g}\left(\nabla_{T Q_{2} X} X, Q_{2} X\right)\right]-\bar{g}\left(\nabla_{T_{2} X}^{s} F Q_{2} X, F Q_{2} X\right) .
\end{aligned}
$$

Since (28) holds for any $X=Y \in \Gamma(D)$ and by taking the covariant derivative with respect to $\bar{\nabla}_{T Q_{2} X}$, we get

$$
\bar{g}\left(\nabla_{T Q_{2} X}^{s} F Q_{2} X, F Q_{2} X\right)=\sin ^{2} \theta\left[g\left(\nabla_{T Q_{2} X} Q_{2} X, Q_{2} X\right)\right] .
$$

Using (51) in (50), we obtain

$$
\cos ^{2} \theta\left[g\left(Q_{2} X, Q_{2} X\right)\right] \bar{g}\left(\alpha_{S}, F Q_{2} X\right)=0
$$

Since $M$ is a proper slant lightlike submanifold and $g$ is a Riemannian metric on $D$, therefore we have $\bar{g}\left(\alpha_{s}, F Q_{2} X\right)=0$. Thus using Lemma 8 and (33), we obtain

$$
\alpha_{S} \in \Gamma(\mu)
$$

Now using the cosymplectic property of $\bar{M}$, we have $\bar{\nabla}_{X} \phi Y=$ $\phi \bar{\nabla}_{X} Y$, for any $X, Y \in \Gamma(D)$, and then using (9), (14), and (29), we obtain

$$
\begin{aligned}
\nabla_{X} T Q_{2} Y+g\left(X, T Q_{2} Y\right) \alpha-A_{F Q_{2} Y} X \\
+\nabla_{X}^{s} F Q_{2} Y+D^{l}\left(X, F Q_{2} Y\right) \\
=T \nabla_{X} Y+F \nabla_{X} Y+g(X, Y) \phi \alpha .
\end{aligned}
$$

Taking the scalar product of both sides of (54), with respect to $\phi \alpha_{S}$ and then using (2), (34) and (53), we obtain

$$
\bar{g}\left(\nabla_{X}^{s} F Q_{2} Y, \phi \alpha_{S}\right)=g\left(Q_{2} X, Q_{2} Y\right) \bar{g}\left(\alpha_{S}, \alpha_{S}\right) .
$$


Since $\mu$ is an invariant subspace, therefore using the cosymplectic character of $\bar{M}$, we have $\bar{\nabla}_{X} \phi \alpha_{S}=\phi \bar{\nabla}_{X} \alpha_{S}$, and this implies that

$$
\begin{gathered}
-A_{\phi \alpha_{S}} X+\nabla_{X}^{s} \phi \alpha_{S}+D^{l}\left(X, \phi \alpha_{S}\right) \\
=-T A_{\alpha_{S}} X-F A_{\alpha_{S}} X+B \nabla_{X}^{s} \alpha_{S} \\
+C \nabla_{X}^{s} \alpha_{S}+\phi D^{l}\left(X, \alpha_{S}\right) .
\end{gathered}
$$

Taking the scalar product of both sides of the previous equation with respect to $F Q_{2} Y$, we obtain

$$
\bar{g}\left(\nabla_{X}^{s} \phi \alpha_{S}, F Q_{2} Y\right)=-\bar{g}\left(F A_{\alpha_{S}} X, F Q_{2} Y\right)+\bar{g}\left(C \nabla_{X}^{S} \alpha_{S}, F Q_{2} Y\right) .
$$

From (15), we know that $B U$ and $C U$ are tangential and transversal components of $\phi(U)$, respectively, for any $U \in$ $\Gamma(\operatorname{tr}(T M))$. Therefore if $U \in \Gamma(\operatorname{ltr}(T M))$, then $\phi U=B U \in$ $\Gamma(\phi \operatorname{ltr}(T M)) \subset S(T M)$ and $C U=0$. Moreover, since $S\left(T M^{\perp}\right)=F(D) \perp \mu$, therefore for any $U \in \Gamma\left(S\left(T M^{\perp}\right)\right)$, $B U \in \Gamma(D) \subset S(T M)$ and $C U \in \Gamma(\mu) \subset \operatorname{tr}(T M)$. Since $\nabla_{X}^{s} \alpha_{S} \in \Gamma\left(S\left(T M^{\perp}\right)\right)$, and therefore $C \nabla_{X}^{s} \alpha_{S} \in \Gamma(\mu)$, and using this with (28), we get

$$
\begin{aligned}
\bar{g}\left(\nabla_{X}^{s} \phi \alpha_{S}, F Q_{2} Y\right) & =-\bar{g}\left(F A_{\alpha_{S}} X, F Q_{2} Y\right) \\
& =-\sin ^{2} \theta\left[g\left(A_{\alpha_{S}} X, Q_{2} Y\right)\right] .
\end{aligned}
$$

Since $\bar{\nabla}$ is a metric connection, therefore $\left(\bar{\nabla}_{X} g\right)\left(F Q_{2} Y, \phi \alpha_{S}\right)=$ 0 . This further implies that $\bar{g}\left(\nabla_{X}^{s} F Q_{2} Y, \phi \alpha_{S}\right)=$ $\bar{g}\left(\nabla_{X}^{s} \phi \alpha_{S}, F Q_{2} Y\right)$, and therefore using (58), we obtain

$$
\bar{g}\left(\nabla_{X}^{s} F Q_{2} Y, \phi \alpha_{S}\right)=-\sin ^{2} \theta\left[g\left(A_{\alpha_{S}} X, Q_{2} Y\right)\right] .
$$

From (55) and (59), we have

$$
g\left(Q_{2} X, Q_{2} Y\right) \bar{g}\left(\alpha_{S}, \alpha_{S}\right)=-\sin ^{2} \theta\left[g\left(A_{\alpha_{S}} X, Q_{2} Y\right)\right]
$$

Then using (10) in (60), we obtain

$$
\begin{aligned}
g\left(Q_{2} X, Q_{2} Y\right) \bar{g}\left(\alpha_{S}, \alpha_{S}\right) & =-\sin ^{2} \theta\left[\bar{g}\left(h^{s}\left(Q_{2} X, Q_{2} Y\right), \alpha_{S}\right)\right] \\
& =-\sin ^{2} \theta\left[g\left(Q_{2} X, Q_{2} Y\right)\right] \bar{g}\left(\alpha_{S}, \alpha_{S}\right),
\end{aligned}
$$

this implies that

$$
\left(1+\sin ^{2} \theta\right)\left[g\left(Q_{2} X, Q_{2} Y\right)\right] \bar{g}\left(\alpha_{S}, \alpha_{S}\right)=0 .
$$

Since $g$ is a Riemannian metric on $D$, thus we obtain

$$
\alpha_{S}=0
$$

Next, for $X \in \Gamma(D)$, using the cosymplectic character of $\bar{M}$, we have $\bar{\nabla}_{X} \phi X=\phi \bar{\nabla}_{X} X$, and this implies that $\nabla_{X} T Q_{2} X+h(X$, $\left.T Q_{2} X\right)-A_{F Q_{2} X} X+\nabla_{X}^{s} F Q_{2} X+D^{l}\left(X, F Q_{2} X\right)=T \nabla_{X} X+$ $F \nabla_{X} X+B h(X, X)+C h(X, X)$. Since $M$ is totally contact umbilical slant lightlike manifold, therefore using
$h\left(X, T Q_{2} X\right)=0$ in the previous equation and then comparing the tangential components, we obtain

$$
\nabla_{X} T Q_{2} X-A_{F_{2} X} X=T \nabla_{X} X+B h(X, X) .
$$

Taking the scalar product of both sides of (64) with respect to $\phi \xi \in \Gamma(\phi \operatorname{Rad}(T M))$ and using Lemma 10, we get

$$
\bar{g}\left(A_{F_{2} X} X, \phi \xi\right)+\bar{g}\left(h^{l}\left(Q_{2} X, Q_{2} X\right), \xi\right)=0
$$

Now using (9), we have

$$
\begin{aligned}
\bar{g} & \left(h^{s}(X, \phi \xi), F Q_{2} X\right)+\bar{g}\left(\phi \xi, D^{l}\left(X, F Q_{2} X\right)\right) \\
& =\bar{g}\left(A_{F Q_{2} X} X, \phi \xi\right)
\end{aligned}
$$

Since $M$ is a totally contact umbilical slant lightlike submanifold therefore using (63) in the previous equation, we obtain

$$
\bar{g}\left(A_{F Q_{2} X} X, \phi \xi\right)=0
$$

Using (67) in (65), we obtain that $\bar{g}\left(h^{l}\left(Q_{2} X, Q_{2} X\right), \xi\right)=0$ and then using (30), we get

$$
g\left(Q_{2} X, Q_{2} X\right) \bar{g}\left(\alpha_{L}, \xi\right)=0
$$

Since $g$ is a Riemannian metric on $D$, therefore $\bar{g}\left(\alpha_{L}, \xi\right)=0$, then using (6), we obtain that

$$
\alpha_{L}=0
$$

Thus from (63) and (69), the proof is complete.

Denote by $\bar{R}$ and $R$ the curvature tensors of $\bar{\nabla}$ and $\nabla$ respectively, and then by straightforward calculations [12], we have

$$
\begin{aligned}
& \bar{R}(X, Y) Z \\
&= R(X, Y) Z+A_{h^{l}(X, Z)} Y-A_{h^{l}(Y, Z)} X+A_{h^{s}(X, Z)} Y \\
&-A_{h^{s}(Y, Z)} X+\left(\nabla_{X} h^{l}\right)(Y, Z)-\left(\nabla_{Y} h^{l}\right)(X, Z) \\
&+D^{l}\left(X, h^{s}(Y, Z)\right)-D^{l}\left(Y, h^{s}(X, Z)\right)+\left(\nabla_{X} h^{s}\right)(Y, Z) \\
&-\left(\nabla_{Y} h^{s}\right)(X, Z)+D^{s}\left(X, h^{l}(Y, Z)\right)-D^{s}\left(Y, h^{l}(X, Z)\right),
\end{aligned}
$$

where

$$
\begin{aligned}
& \left(\nabla_{X} h^{s}\right)(Y, Z)=\nabla_{X}^{s} h^{s}(Y, Z)-h^{s}\left(\nabla_{X} Y, Z\right)-h^{s}\left(Y, \nabla_{X} Z\right) \\
& \left(\nabla_{X} h^{l}\right)(Y, Z)=\nabla_{X}^{l} h^{l}(Y, Z)-h^{l}\left(\nabla_{X} Y, Z\right)-h^{l}\left(Y, \nabla_{X} Z\right) .
\end{aligned}
$$


An indefinite cosymplectic space form is a connected indefinite cosymplectic manifold of constant holomorphic sectional curvature $c$ and is denoted by $\bar{M}(c)$. Then the curvature tensor $\bar{R}$ of $\bar{M}(c)$ is given by

$$
\begin{aligned}
& \bar{R}(X, Y) Z \\
&=\frac{c}{4}\{\bar{g}(Y, Z) X-\bar{g}(X, Z) Y+\eta(X) \eta(Z) Y \\
& \quad-\eta(Y) \eta(Z) X+\bar{g}(X, Z) \eta(Y) V-g(Y, Z) \eta(X) V \\
&\quad+\bar{g}(\phi Y, Z) \phi X+\bar{g}(\phi Z, X) \phi Y-2 \bar{g}(\phi X, Y) \phi Z\}
\end{aligned}
$$

for $X, Y, Z$ vector fields on $\bar{M}$.

Theorem 12. There do not exist totally contact umbilical proper slant lightlike submanifolds of an indefinite cosymplectic space form $\bar{M}(c)$ such that $c \neq 0$.

Proof. Suppose $M$ be a totally contact umbilical proper lightlike submanifold of $\bar{M}(c)$ such that $c \neq 0$. Then using (73), for any $X \in \Gamma(D), Z \in \Gamma(\phi \operatorname{ltr}(T M))$, and $\xi \in \Gamma(\operatorname{Rad}(T M))$, we obtain

$$
\bar{g}(\bar{R}(X, \phi X) Z, \xi)=-\frac{c}{2} g(\phi X, \phi X) \bar{g}(\phi Z, \xi) .
$$

Then using (2), we get

$$
\bar{g}(\bar{R}(X, \phi X) Z, \xi)=-\frac{c}{2} g\left(Q_{2} X, Q_{2} X\right) \bar{g}(\phi Z, \xi) .
$$

On the other hand using (31) and (63) in (70), we get

$$
\begin{aligned}
\bar{g}(\bar{R}(X, \phi X) Z, \xi)= & \bar{g}\left(\left(\nabla_{X} h^{l}\right)(\phi X, Z), \xi\right) \\
& -\bar{g}\left(\left(\nabla_{\phi X} h^{l}\right)(X, Z), \xi\right) .
\end{aligned}
$$

Using (30) and (72), we have

$$
\left(\nabla_{X} h^{l}\right)(\phi X, Z)=-g\left(\nabla_{X} T Q_{2} X, Z\right) \alpha_{L}-g\left(T Q_{2} X, \nabla_{X} Z\right) \alpha_{L}
$$

Similarly

$$
\left(\nabla_{\phi X} h^{l}\right)(X, Z)=-g\left(\nabla_{\phi X} X, Z\right) \alpha_{L}-g\left(X, \nabla_{\phi X} Z\right) \alpha_{L}
$$

Using (77) and (78) in (76), we obtain

$$
\begin{aligned}
\bar{g}( & \bar{R}(X, \phi X) Z, \xi) \\
= & -g\left(\nabla_{X} T Q_{2} X, Z\right) \bar{g}\left(\alpha_{L}, \xi\right)-g\left(T Q_{2} X, \nabla_{X} Z\right) \bar{g}\left(\alpha_{L}, \xi\right) \\
& +g\left(\nabla_{\phi X} X, Z\right) \bar{g}\left(\alpha_{L}, \xi\right)+g\left(X, \nabla_{\phi X} Z\right) \bar{g}\left(\alpha_{L}, \xi\right)
\end{aligned}
$$

Now using (69), we have

$$
\bar{g}(\bar{R}(X, \phi X) Z, \xi)=0
$$

Thus using (80) in (75), we have

$$
\frac{c}{2} g\left(Q_{2} X, Q_{2} X\right) \bar{g}(\phi Z, \xi)=0 .
$$

Since $g$ is a Riemannian metric on $D$ and (81) implies that $\bar{g}(\phi Z, \xi) \neq 0$, therefore $c=0$. This contradiction completes the proof.

In [12], a minimal lightlike submanifold $M$ is defined when $M$ is a hypersurface of a 4-dimensional Minkowski space. Then in [15], a general notion of minimal lightlike submanifold of a semi-Riemannian manifold $\bar{M}$ is introduced as follows.

Definition 13. A lightlike submanifold $(M, g, S(T M))$ isometrically immersed in a semi-Riemannian manifold $(\bar{M}, \bar{g})$ is minimal if

(i) $h^{s}=0$ on $\operatorname{Rad}(T M)$,

(ii) trace $h=0$, where trace is written with respect to $g$ restricted to $S(T M)$.

We use the quasi-orthonormal basis of $M$ given by

$$
\left\{\xi_{1}, \ldots, \xi_{r}, \phi \xi_{1}, \ldots, \phi \xi_{r}, V, e_{1}, \ldots, e_{q}, \phi N_{1}, \ldots, \phi N_{r}\right\},
$$

such that $\left\{\xi_{1}, \ldots, \xi_{r}\right\},\left\{\phi \xi_{1}, \ldots, \phi \xi_{r}\right\},\left\{e_{1}, \ldots, e_{q}\right\}$, and $\left\{\phi N_{1}\right.$, $\left.\ldots, \phi N_{r}\right\}$ form a basis of $\operatorname{Rad}(T M), \phi(\operatorname{Rad}(T M)), D$, and $\phi(\operatorname{ltr}(T M))$, respectively.

Definition 14 (see [16]). A lightlike submanifold is called irrotational if and only if $\bar{\nabla}_{X} \xi \in \Gamma(T M)$ for all $X \in \Gamma(T M)$ and $\xi \in \Gamma(\operatorname{Rad}(T M))$.

Theorem 15. Let $M$ be an irrotational slant lightlike submanifold of an indefinite cosymplectic manifold $\bar{M}$. Then $M$ is minimal if and only if

$$
\left.\operatorname{trace} A_{W_{k}}\right|_{S(T M)}=0,\left.\quad \operatorname{trace} A_{\xi_{i}}^{*}\right|_{S(T M)}=0,
$$

where $\left\{W_{k}\right\}_{k=1}^{l}$ is a basis of $S\left(T M^{\perp}\right)$ and $\left\{\xi_{i}\right\}_{i=1}^{r}$ is a basis of $\operatorname{Rad}(T M)$.

Proof. Since $\bar{\nabla}_{V} V=0$, therefore using (8), we get $h^{l}(V, V)=0$ and $h^{s}(V, V)=0$. Moreover, irrotational $M$ implies $h^{s}(X, \xi)=$ 0 for $X \in \Gamma(T M)$ and $\xi \in \Gamma(\operatorname{Rad}(T M))$. Thus $h^{s}$ vanishes on $\operatorname{Rad}(T M)$. Hence $M$ is minimal if and only if trace $h=0$ on $S(T M)$; that is, $M$ is minimal if and only if

$$
\sum_{i=1}^{r} h\left(\phi \xi_{i}, \phi \xi_{i}\right)+\sum_{i=1}^{r} h\left(\phi N_{i}, \phi N_{i}\right)+\sum_{j=1}^{q} h\left(e_{j}, e_{j}\right)=0 .
$$

Using (10) and (12), we obtain

$$
\begin{aligned}
\sum_{i=1}^{r} h\left(\phi \xi_{i}, \phi \xi_{i}\right)=\sum_{i=1}^{r}\left\{\frac{1}{r} \sum_{a=1}^{r} \bar{g}\left(A_{\xi_{a}}^{*} \phi \xi_{i}, \phi \xi_{i}\right) N_{a}\right. \\
\left.+\frac{1}{l} \sum_{k=1}^{l} \bar{g}\left(A_{W_{k}} \phi \xi_{i}, \phi \xi_{i}\right) W_{k}\right\} .
\end{aligned}
$$


Similarly, we have

$$
\begin{aligned}
& \sum_{i=1}^{r} h\left(\phi N_{i}, \phi N_{i}\right)=\sum_{i=1}^{r}\left\{\frac{1}{r} \sum_{a=1}^{r} g\left(A_{\xi_{a}}^{*} \phi N_{i}, \phi N_{i}\right) N_{a}\right. \\
&\left.+\frac{1}{l} \sum_{k=1}^{l} g\left(A_{W_{k}} e_{j}, e_{j}\right) W_{k}\right\}, \\
& \sum_{j=1}^{q} h\left(e_{j}, e_{j}\right)=\sum_{j=1}^{q}\left\{\frac{1}{r} \sum_{i=1}^{r} g\left(A_{\xi_{i}}^{*} e_{j}, e_{j}\right) N_{i}\right. \\
&\left.+\frac{1}{l} \sum_{k=1}^{l} g\left(A_{W_{k}} e_{j}, e_{j}\right) W_{k}\right\} .
\end{aligned}
$$

Thus our assertion follows from (85) and (86).

\section{References}

[1] B.-Y. Chen, "Slant immersions," Bulletin of the Australian Mathematical Society, vol. 41, no. 1, pp. 135-147, 1990.

[2] B.-Y. Chen, Geometry of Slant Submanifolds, Katholieke Universiteit Leuven, Louvain, Belgium, 1990.

[3] A. Lotta, "Slant submanifolds in contact geometry," Bulletin Mathématique de la Société des Sciences Mathématiques de Roumanie, vol. 39, pp. 183-198, 1996.

[4] A. Lotta, "Three-dimensional slant submanifolds of $K$-contact manifolds," Balkan Journal of Geometry and Its Applications, vol. 3, no. 1, pp. 37-51, 1998.

[5] J. L. Cabrerizo, A. Carriazo, L. M. Fernández, and M. Fernández, "Slant submanifolds in Sasakian manifolds," Glasgow Mathematical Journal, vol. 42, no. 1, pp. 125-138, 2000.

[6] J. L. Cabrerizo, A. Carriazo, L. M. Fernández, and M. Fernández, "Semi-slant submanifolds of a Sasakian manifold," Geometriae Dedicata, vol. 78, no. 2, pp. 183-199, 1999.

[7] B. Şahin, "Slant lightlike submanifolds of indefinite Hermitian manifolds," Balkan Journal of Geometry and Its Applications, vol. 13, no. 1, pp. 107-119, 2008.

[8] R. S. Gupta, A. Upadhyay, and A. Sharfuddin, "Slant lightlike submanifolds of indefinite cosymplectic manifolds," Mediterranean Journal of Mathematics, vol. 8, no. 2, pp. 215-227, 2011.

[9] V. Jain, R. Kumar, and R. K. Nagaich, "Non existence of totally contact umbilical GCR-lightlike submanifolds of indefinite cosymplectic manifolds," Vietnam Journal of Mathematics, 2013.

[10] R. Kumar, R. Rani, and R. K. Nagaich, "On sectional curvatures of $\epsilon$-Sasakian manifolds," International Journal of Mathematics and Mathematical Sciences, vol. 2007, Article ID 93562, 8 pages, 2007.

[11] D. E. Blair, Riemannian Geometry of Contact and Symplectic Manifolds, vol. 203 of Progress in Mathematics, Birkhäuser, Boston, Mass, USA, 2002.

[12] K. L. Duggal and A. Bejancu, Lightlike Submanifolds of SemiRiemannian Manifolds and Applications, vol. 364 of Mathematics and Its Applications, Kluwer Academic Publishers, Dordrecht, The Netherlands, 1996.

[13] B. O'Neill, Semi-Riemannian Geometry with Applications to Relativity, vol. 103 of Pure and Applied Mathematics, Academic Press, New York, NY, USA, 1983.

[14] K. Yano and M. Kon, Structures on Manifolds, vol. 3 of Series in Pure Mathematics, World Scientific Publishing, Singapore, 1984.
[15] C. L. Bejan and K. L. Duggal, "Global lightlike manifolds and harmonicity," Kodai Mathematical Journal, vol. 28, no. 1, pp. 131145, 2005.

[16] K. L. Duggal and B. Sahin, "Screen Cauchy Riemann lightlike submanifolds," Acta Mathematica Hungarica, vol. 106, no. 1-2, pp. 137-165, 2005. 


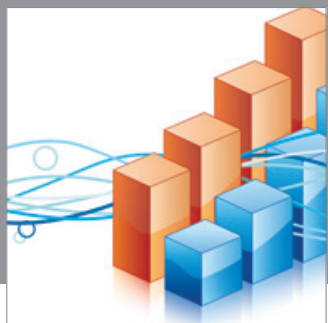

Advances in

Operations Research

mansans

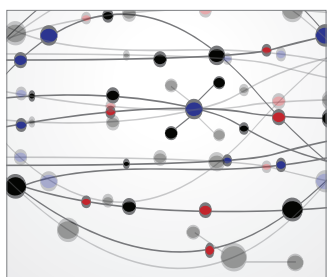

The Scientific World Journal
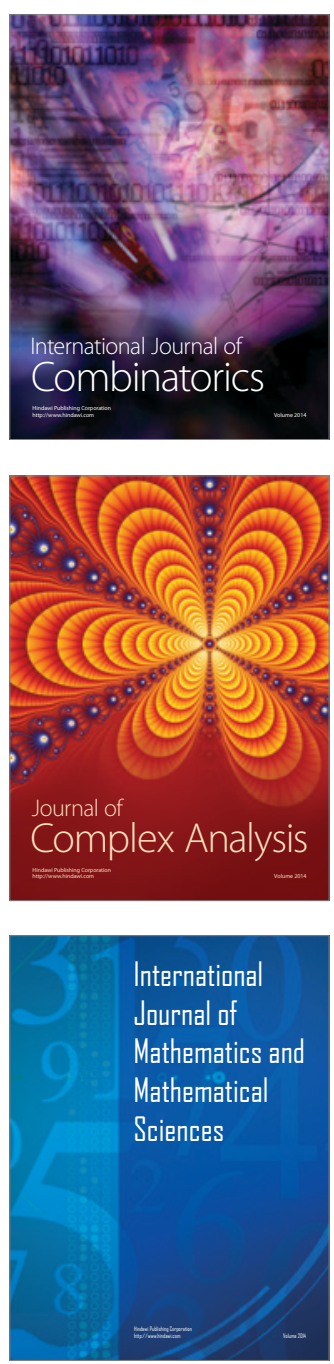
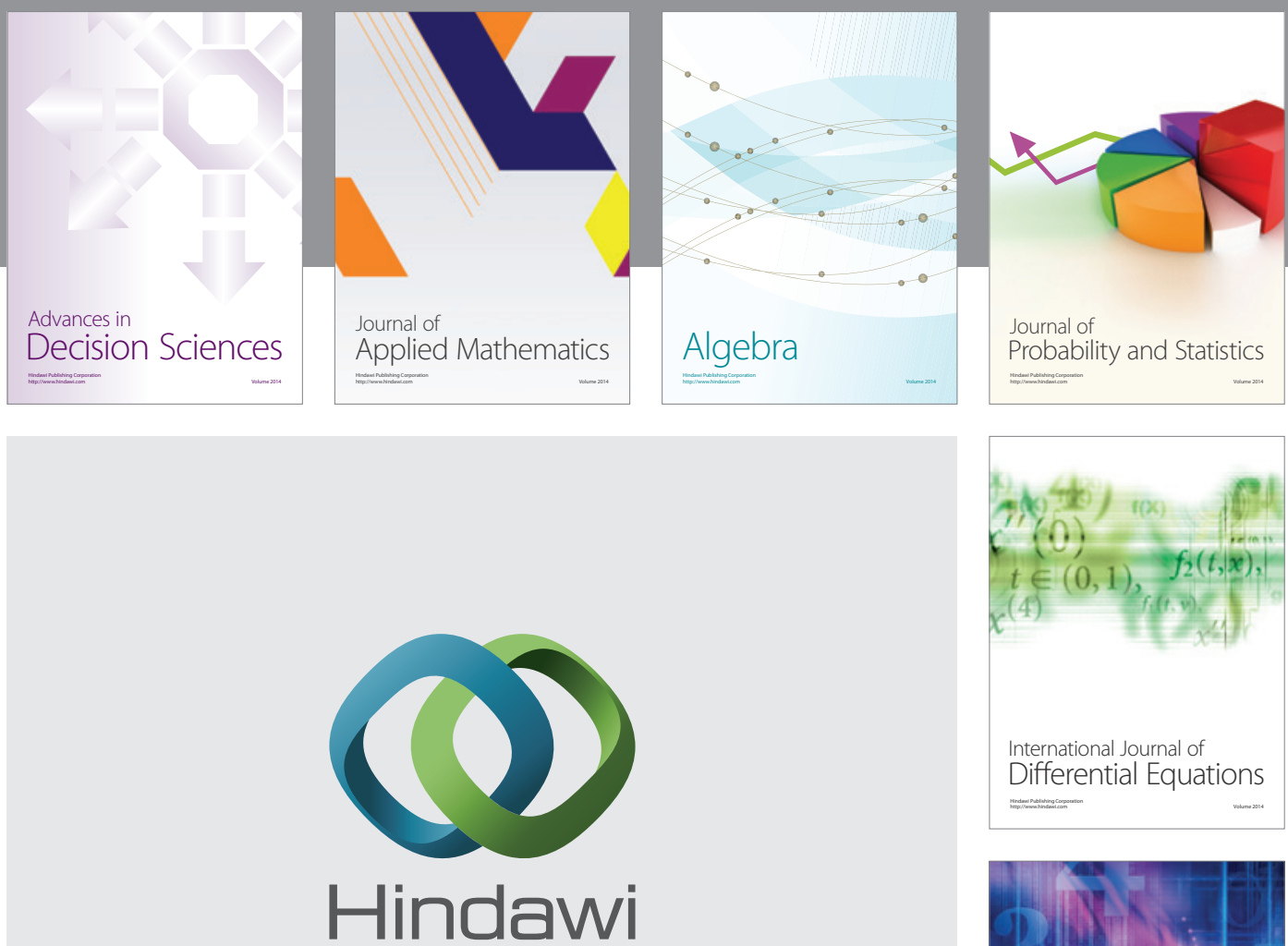

Submit your manuscripts at http://www.hindawi.com
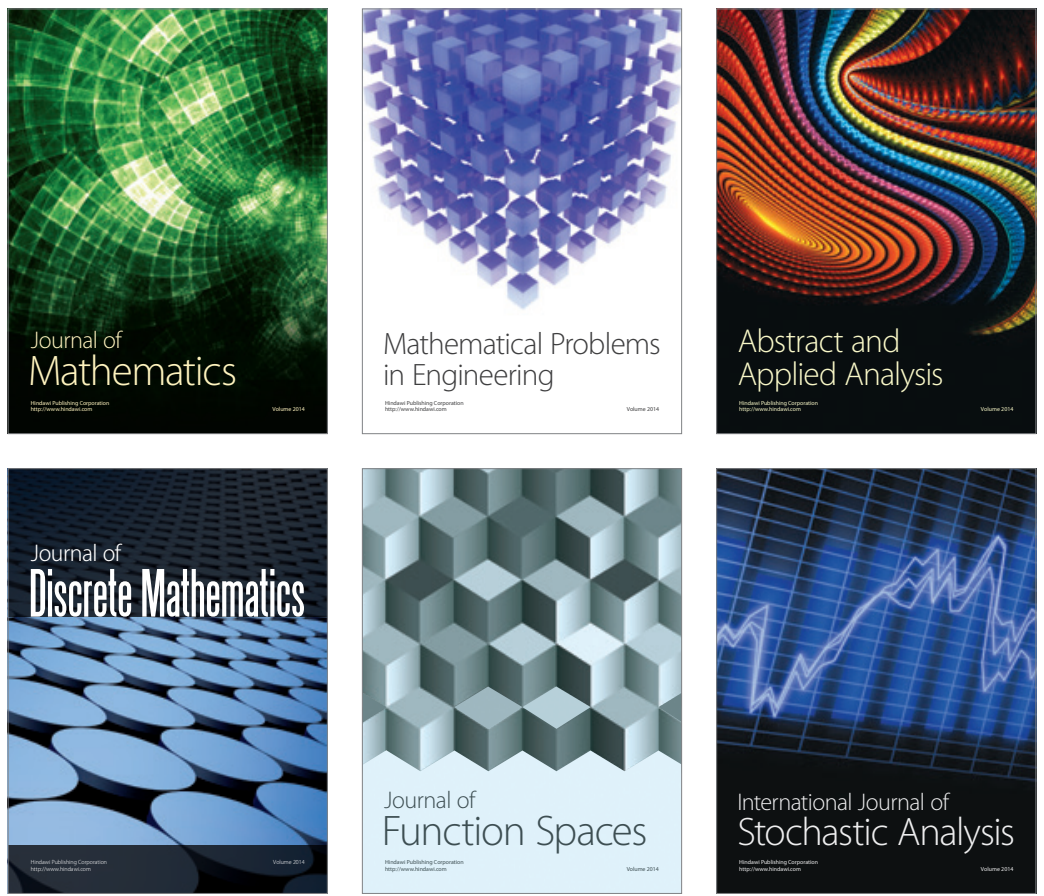

Journal of

Function Spaces

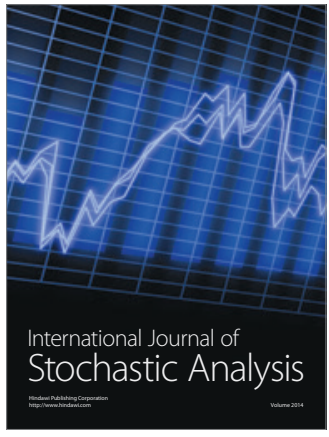

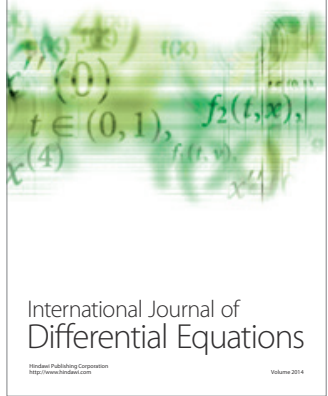
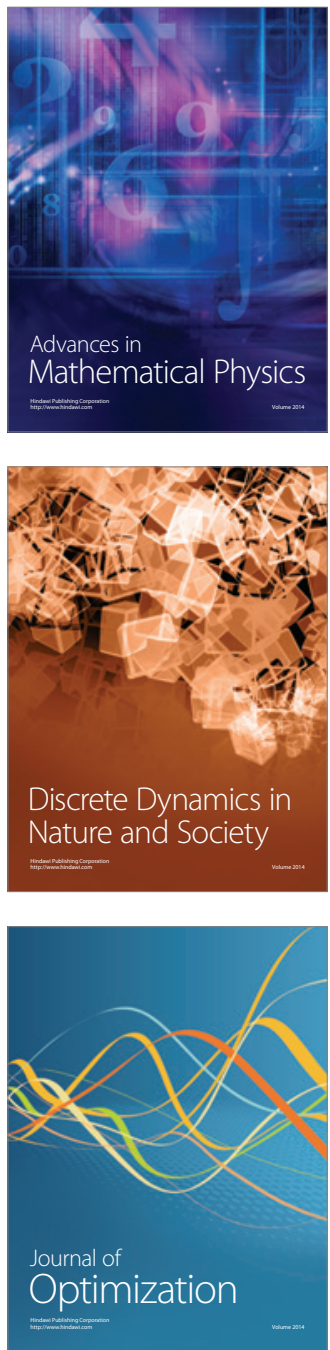